\title{
Ascorbic Acid Deficiency Reduces the Level of mRNA for Cytochrome P-450 on the Induction by Polychlorinated Biphenyls
}

\author{
Natsuki Matsushita, Tomoko Kobayashi, ${ }^{1}$ Hiroaki OdA, \\ Fumihiko Horio, and Akira YoshIDA \\ Loboratory of Nutritional Biochemistry, Department of Agricultural Chemistry, \\ Nagoya University, Nagoya 464-01, Japan \\ ${ }^{1}$ Fuculty of Pharmaceutical Sciences, Nagoya City University, Nagoya 467, Japan
}

(Received January 11, 1993)

\begin{abstract}
Summary Ascorbic acid (AsA) deficiency causes a decrease in hepatic concentration of cytochrome P-450 and a decrease in hepatic activity of drug-metabolizing enzymes in rats unable to synthesize AsA (ODS rats). To study the mechanism of the decrease in hepatic concentration of cytochrome P-450 isozymes by AsA deficiency, we chose the xenobioticsinducible cytochrome P-450 and performed the experiments indicated below. AsA-deficient rats were fed polychlorinated biphenyls (PCB) which markedly induce both CYP1A subfamily and several isozymes in CYP2B subfamily. First, we assayed the activities of two drugmetabolizing enzymes so that one could be functionally distinguished from another. AsA deficiency significantly reduced the hepatic activity of aminopyrine-N-demethylase in ODS rats with and without dietary PCB, but had no effect on benzo(a)pyrene hydroxylase activity. Secondly, quantitative immunoblot analyses demonstrated that the levels of CYP2B1/2B2 and CYP1A1 in the AsA-deficiency rats fed PCB were approximately 60 and $80 \%$ lower than those found in rats fed AsAsupplemented diet. The degree of reduction in CYP2B1/2B2 was greater than CYP1A1. Thirdly, AsA deficiency caused a decrease in hepatic abundance of CYP2B1/2B2 mRNA, whereas it had no effect on the levels of CYP1A1 and 1A2 mRNA. These results indicated that dietary AsA selectively affects the levels of CYP2B1/2B2 mRNA among cytochrome P-450 induced by PCB and plays important roles for optimum induction of drug-inducible cytochrome P-450. We concluded that AsA deficiency decreases specific froms of drug-inducible cytochrome P-450, especially CYP2B1/2B2 and that the reduction of CYP2B1/2B2 mRNA level in AsA-deficient rats caused a decrease in cytochrome P-450 concentration and hepatic activity of drug-metabolizing enzymes.
\end{abstract}


Key Words L-ascorbic acid, cytochrome P-450, drug-metabolizing enzyme, ODS rats

Cytochrome P-450 are a large family of hemoproteins that function in the metabolism of endogenous and exogenous compounds, including steroid hormones and xenobiotics (1-3). Previous studies demonstrated that the cytochrome P-450dependent mixed function monooxygenases (MFO) are affectecd by nutritional conditions, including L-ascorbic acid (AsA) as well as several other factors such as sex and age $(4,5)$. It is established that AsA deficiency significantly reduces the concentration of cytochrome P-450 and the activities of drug-metabolizing enzymes in liver microsomes (6-9). Numerous studies have been made on the physiological function of AsA. For example, one of the essential functions of AsA may be a role as cofactor for several physiological hydroxylations (10). We and many investigators have studied the nutritional significance of AsA for the maintenance and induction of MFO-related cytochrome P-450 (6-9). Horio et al. (9) reported the reduction of the activities of MFO in AsA deficiency, using Osteogenic-DisorderShionogi rat (ODS rats), a mutant unable to synthesize AsA (11). On the other hand, various xenobiotics have been shown to enhance markedly the biosynthesis of AsA in rats $(12,13)$, and to increase tissue levels of AsA in liver, kidney, and muscle (12). In addition, Horio et al. (9) indicated that AsA-requirement for maximal induction of cytochrome P-450 and MFO by xenobiotics is higher than for normal growth in an ODS rat.

As mentioned above, such information is very suggestive that AsA plays an important role in the maintenance and induction of cytochrome P-450. The biochemical mechanism by which AsA affects the cytochrome P-450, however, is not clear. AsA deficiency decreased the concentration of total cytochrome P-450, but even in a severely AsA-deficiency status the concentration of total cytochrome P-450 was only $40 \%$ of the normal level. Zannoni et al. $(6,7)$ showed that the activities of drug-metabolizing enzymes were affected to different extents by AsA deficiency. Kanazawa et al. (14) also showed that AsA deficiency decreased specific forms of cytochrome P-450 in liver microsomes of guinea pigs. These observations led us to postulate that AsA deficiency might differentially affect individual forms of cytochrome P-450.

The effects of AsA on total amounts of cytochrome P-450 have been studied, but the exact role of AsA in the induction of individual form of cytochrome P-450 by xenobiotics still remains to be elucidated. The xenobiotics-inducible forms of cytochrome P-450 can be divided into two types, methylchoranthrene (MC)inducible forms (CYP1A1 and CYP1A2) and phenobarbital (PB)-inducible forms (CYP2B1 and CYP2B2). They catalyze the oxidative metabolism of a wide variety of exogenous compounds, for example, MC-inducible forms catalyze a benzo(a) pyrene hydroxylase and PB-inducible forms catalyze an aminopyrine-Ndemethylase and so on. Each of the two MFO activities is catalyzed by a distinct form of cytochrome P-450. In the present study, therefore, in order to investigate 
the effect of AsA on a specific form of xenobiotics-inducible cytochrome P-450, we studied the effect of AsA deficiency on the two MFO activities and the hepatic level of mRNA for the two types of cytochrome P-450 in ODS rats. In this study we used polychlorinated biphenyls (PCB) that markedly induce both of the two types of xenobiotics-inducible cytochrome P-450.

Our present study showed that aminopyrine-N-demethylase activity and the level of CYP2B1/2B2 mRNA induced by PCB were lower in AsA deficiency than those in ODS rats fed a diet supplemented with AsA, and that AsA deficiency affected neither benzo(a)pyrene hydroxylase activity nor the level of CYP1A1 and 1A2 mRNA. From these results we demonstrated that AsA might differentially affect the induction of individual forms of xenobiotics-inducible cytochrome P-450.

\section{EXPERIMENTAL}

Animals and diets. Male ODS-od/od mutant rats unable to synthesize AsA were obtained from Japan Clea, Osaka. Five-week-old ODS rats weighing about 80 $\mathrm{g}$ were used. The composition ( $\mathrm{g} / \mathrm{kg}$ diet) of a basal diet was as follows: casein, 250; AIN76 mineral mixture (15), 35; corn oil, 50; AIN76A vitamin mixture (16), 10; choline chloride, 2; cellulose powder (AVICEL type FD-101, Asahi Chemical Industry Co., Tokyo), 50; a mixture of sucrose and cornstarch $(1: 2), 603$. The vitamin mixture did not contain L-ascorbic acid. Before the experiment ODS rats were fed a bassl diet supplemented with $300 \mathrm{mg}$ L-ascorbic acid per kilogram diet for 7 days. Rats were kept at $24^{\circ} \mathrm{C}$ with a $12 \mathrm{~h}$ cycle of light (from $0800 \mathrm{~h}$ to 2000 h) and dark $(2000 \mathrm{~h}-0800 \mathrm{~h})$. All rats were individually housed in wire screenbottomed cages, and provided food and water ad libitum except for some in group $\mathrm{C}$ and $\mathrm{F}$ (see below).

Experimental schedule. Rats were divided into six groups of four rats each (Table 1). Each group was fed test diets as follows for 15 days. Group A rats were fed the bassal diet. Then, $300 \mathrm{mg}$ AsA (group B), $200 \mathrm{mg}$ PCB (Aroclor 1254, Mitsubishi Monsanto Co., Ltd., Tokyo) (group D) or $300 \mathrm{mg}$ AsA and $200 \mathrm{mg}$ PCB simultaneously (group E) were added per kilogram of the basal diet in place of carbohydrate. Group A, B, D and E rats were fed these test diets ad libitum for experimental 15 day period. Group $\mathrm{C}$ and $\mathrm{F}$ rats were fed the test diets similar to group $A$ and $D$, respectively. Group $C$ and $F$ rats were fed ad libitum to day 10 , and but from day 11 to the end of the experimental period were pair-fed to the mean of food intake in group A and D, respectively. We previously observed that body weight in ODS rats fed AsA-free diet began to decrease at 10-12 days due to decreasing food intake (17). Rats were killed by decapitation between $1000 \mathrm{~h}$ and $1100 \mathrm{~h}$ on day 15 . Liver was quickly removed, weighed, and hepatic microsomal fraction was prepared as described previously (18) for assay of the amount of cytochrome P-450 and the activities of several drug-metabolizing enzymes. Total RNA was prepared from a portion of liver (see below).

Analytical methods. Amount of cytochrome P-450 and the activity of hepatic 
Table 1. Group designation, experimental diet, and liver levels of ascorbic acid in rats fed ascorbic acid-free diet and/or dietary PCB. ${ }^{1,2}$

\begin{tabular}{|c|c|c|c|c|}
\hline \multirow{2}{*}{$\begin{array}{c}\text { Group } \\
\text { designation }\end{array}$} & \multicolumn{3}{|c|}{ Experimental diet } & \multirow{2}{*}{$\begin{array}{c}\text { Liver ascorbic } \\
\text { acid } \\
(\mu \mathrm{g} / \mathrm{g} \text { liver })\end{array}$} \\
\hline & $\begin{array}{l}\text { L-Ascorbic acid } \\
\text { (mg/kg diet) }\end{array}$ & $\begin{array}{c}\text { PCB } \\
\text { (mg/kg diet) }\end{array}$ & Feeding & \\
\hline A & 0 & 0 & Ad libitum & $14.6 \pm 0.9$ \\
\hline $\mathbf{B}$ & 300 & 0 & Ad libitum & $166 \pm 5.4$ \\
\hline $\mathrm{C}$ & 300 & 0 & Pair-feeding & $140 \pm 3.1$ \\
\hline D & 0 & 200 & Ad libitum & $14.5 \pm 1.3$ \\
\hline $\mathbf{E}$ & 300 & 200 & Ad libitum & $142 \pm 8.3^{*}$ \\
\hline \multirow[t]{4}{*}{$\mathrm{F}$} & 300 & 200 & Pair-feeding & $113 \pm 4.5^{\# \#}$ \\
\hline & & \multicolumn{3}{|c|}{ ANOVA } \\
\hline & & & PCB & $p<0.01$ \\
\hline & & & Interaction & $p<0.05$ \\
\hline
\end{tabular}

\footnotetext{
${ }^{1}$ Values are $\mathbf{M} \pm$ SEM for 4 rats. ${ }^{2}$ Statistical significance of differences among values was analyzed by two-way analysis of variance (ANOVA). Since the interaction (ascorbic acid $\times$ PCB) was significant, Student's $t$-test was performed. ${ }^{*}$ These values differed significantly $(p<0.05)$ from the values of B group using Student's $t$-test. \# These values differed significantly $(p<0.01)$ from the values of $\mathrm{C}$ group using Student's $t$-test.
}

drug-metabollizing enzymes: Hepatic microsomal fraction was prepared as described previously (18). The concentration of hepatic total cytochrome P-450 was determined by the method of Omura and Sato (19). The activity of aminopyrineN-demethylase (EC 1.14.14.1) was measured by the determination of the amount of formaldehyde produced during the incubation (20). Formaldehyde was measured according to the method of Nash (20). Benzo(a)pyrene hydroxylase activity (EC 1.14.14.2) was assayed by the method on Nebert and Gelboin (21). Fluorescence of hydroxylated products was determined in $1 \mathrm{mM} \mathrm{NaOH}$ using a FP-550 spectrofluorometer (Japan Spectoroscopic Co. Ltd.) (excitation $396 \mathrm{~nm}$, emission $522 \mathrm{~nm})$.

Western blot analysis: For western blot analysis of individual microsomal P450s, prepared liver microsomes were electrophoresed on SDS polyacrylamide gels, transferred to Immobilon-P polyvinylidene difluoride (PVDF) membrane (Nihon Milipore Ltd., Tokyo, Japan) and then immunoblotted with anti-cytochrome P-450 antibodies (see below) using protein detection kit (ECL western blotting detection system, Amersham Japan, Tokyo) for detection. According to protocols in this kit, individual P-450 protein levels were determined. Antibodies to CYP1A1 and CYP 2B1 (rabbit anti-rat CYP1A1 and CYB2B1) were purchased from Oxygene Dallas, Texas. SDS polyacrylamide gel electrophoresis was performed by the method of Laemmli (22). For quantitation, visualized membranes were scanned with DualWavelength TLC Scanner CS-910 (Shimadzu Ltd., Kyoto, Japan). 
Isolation of RNA and RNA blot analysis: RNA was extracted from rat liver as described by Chomozynski and Sacchi (23). RNA blot analysis was performed as described by Thomas (24). Total RNA $(10 \mu \mathrm{g})$ was separated by electrophoresis on a $1 \%$ agarose gel containing $6.6 \%$ formaldehyde with $40 \mathrm{~mm} 3-(\mathrm{N}$-morpholino) propanesulfonic acid (MOPS) buffer ( $\mathrm{pH} 7.0$ ), $10 \mathrm{~mm}$ sodium acetate, and $1 \mathrm{mM}$ EDTA. The RNA samples were denatured at $55^{\circ} \mathrm{C}$ for $15 \mathrm{~min}$ in the above MOPS buffer containing $50 \%$ formamide and $6.6 \%$ formaldehyde. The electrophoresis buffer was $40 \mathrm{~mm}$ MOPS (pH 7.0), $10 \mathrm{~mm}$ sodium acetate and $1 \mathrm{~mm}$ EDTA. After electrophoresis, the RNA was transferred directly to nitrocellulose membrane (BAS85 transfer membrane, Schleicher \& Schuell, Germany) in $10 \times$ SSC $(1 \times$ SCC is $0.15 \mathrm{mM} \mathrm{NaCl}$ and $0.015 \mathrm{~mm}$ sodium citrate, $\mathrm{pH} 7.0$ ) by the method of Southern (25). The membrane was baked at $80^{\circ} \mathrm{C}$ for $2 \mathrm{~h}$, and then hybridized with probes for CYP1A2, CYP2B1, or albumin mRNA. Enzymatic labeling of probes, hybridization and detection were perfomed using ECL gene detection system (Amersham Japan, Tokyo). The method depended of the protocol in this detection kit. Slot blot was made with RNA using a Milliblot-S slot blotter. Baking, hybridization and detection were performed as described above. Detection films by slot blot were scanned with Dual-Wavelength TLC Scanner CS-910 (Shimadzu Ltd., Kyoto, Japan), and the hepatic mRNA level was quantitated.

cDNA probe for RNA blot analysis: A rat CYP1A2 (cytochrome P-450d) cDNA plasmid, pcP-450mc-3 (26), was a gift from Dr. K. Kawajiri (Saitama Cancer Center Research Institute, Japan). A PstI-TaqI 331 base pairs long fragment of pcP-450mc-3, in which the homology of nucleotide sequence between CYP1A1 and CYP1A2 is 93.7\%, was used as probe (27). A rat CYP2B1 (cytochrome P-450b) cDNA plasmid, pcP-450pb-4 (28), was a gift from Dr. Y. Fujii-Kuriyama (Tohoku University, Japan). A rat albumin cDNA plasmid, pIKI (29) was a gift from Dr. K. Nakamura (Nagoya University, Japan).

Statistical analysis: Statistical significance of difference among values was analyzed by two-way analyses of variance (ANOVA). When interaction (AsA $\times$ PCB) was significant, Student's $t$-test or Duncan's multiple range test were performed. The criterion for significance was a $p$-value of less than 0.05 .

\section{RESULTS}

Feeding AsA-free diet to ODS rats for 15 days (group A and D) lowered final body weight gain, and restriction of food intake (group C and F) also equivalently

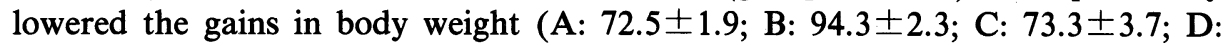
67.3土1.9; E: $96.3 \pm 0.3$; F: 74.8 \pm 0.2 ; g/15 days; $\mathrm{M} \pm$ SEM, ANOVA; AsA-effect: $p<0.01$; PCB-effect: $p>0.05$; interaction: $p>0.05$ ). Body weight in AsA-deficient rats began to decrease on day 11. On the other hand, feeding PCB for 15 days did not affect the gain in body wight. This observation corresponded with the previous report by Horio et al. (17). Table 1 shows liver AsA concentrations in rats fed AsA-deficient diet and/or PCB-supplement diet. Rats fed AsA-free diet (group A 

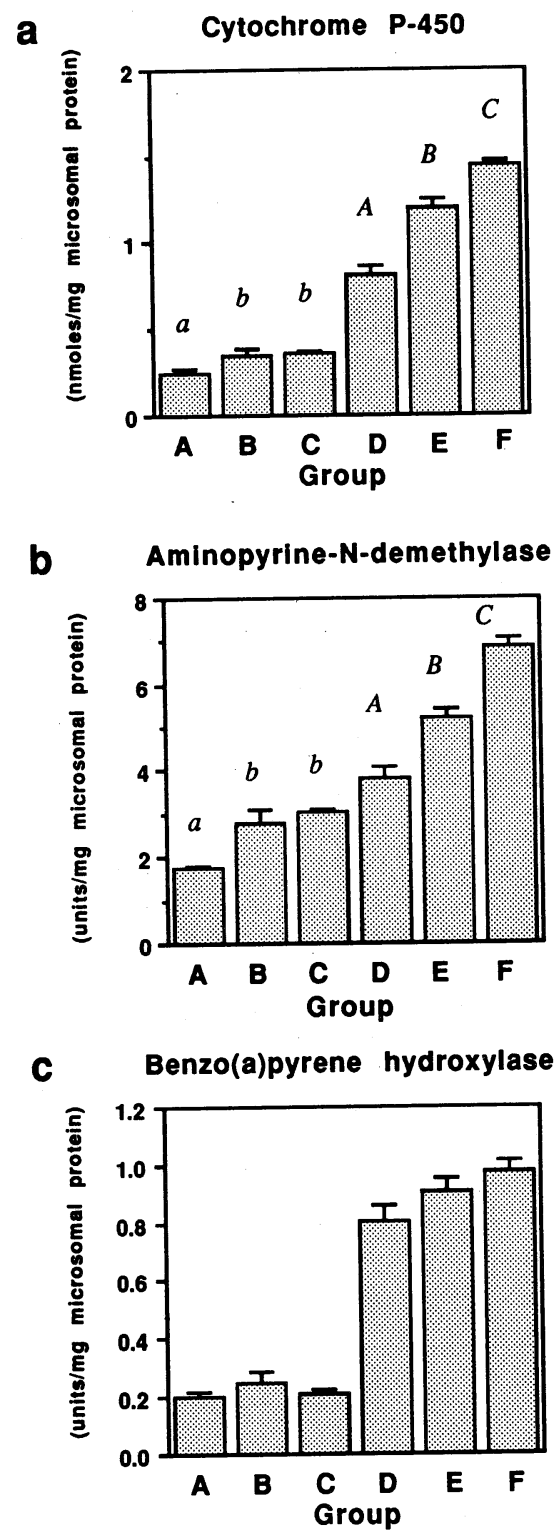

Fig. 1. The concentration of hepatic microsomal total cytochrome P-450 (a) and the activities of drug-metabolizing enzymes (b and $c$ ) in AsA-defeficient rats fed diets with or without PCB. a: The concentration of cytochrome P-450 is expressed as nmoles per milligram microsomal protein. b: Aminopyrine-Ndemethylase activity, the activity is expressed as nmoles of formaldehyde produced per minute per milligram microsomal protein. c: Benzo(a)pyrene hydroxylase activity; the activity is expressed as nmoles of hydroxybenzo(a) 
and D) had significantly lower liver AsA concentrations which were approximately $10 \%$ of control. Feeding PCB also lowered liver AsA level. The interaction of AsA $\times$ PCB was significant and the decrease of liver AsA level by dietary PCB was only significant in rats fed AsA-supplement diet.

Figure 1 shows the concentration of total cytochrome P-450 and the activities of several drug-metabolizing enzymes in liver microsomes of AsA-deficient rats. Both the concentration of total cytochrome P-450 and the specific activities of two drug-metabolizing enzymes measured in this study were induced by deitary PCB. The concentration of cytochrome P-450 was decreased by AsA deficiency in rats with and without dietary PCB (Fig. 1a). Only in groups fed PCB did pair-feeding increase cytochrome P-450 more than by feeding ad libitum (compare E with F) (Fig. 1a). Liver microsomal activities of aminopyrine-N-demethylase were also lower in AsA deficiency by approximately 60\% as observed in the change of cytochrome P-450 (Fig. 1b). This reduction was observed in both rats with and without dietary PCB. On the other hand, AsA deficiency had no effect on benzo(a) pyrene hydroxylase activitiy (Fig. 1c). As mentioned above, dietary AsA differntially affected the activity of individual drug-metabolizing enzymes.

As shown in Fig. 2, the amounts of each form of cytochrome P-450 induced by PCB were quantitated by western blot analysis using the specific antibodies to rat CYP1A1 and CYP2B1. According to the information in the blotting kit used in this study (Oxygene Dallas Co., Ltd., Texas), the antibody to CYP1A1 used in this study recognizes a single microsomal protein, and the antibody to CYP2B1 was cross-reactive with CYP2B2. The intensities of stained bands of group D, E, and $F$ treated with $\mathrm{PCB}$ were calculated and expressed in arbitrary relative units (Fig. 2c, d). In AsA deficiency, the amounts of cytochrome P-450 immunochemically related to CYP1A1 and CYP2B1 were decreased approximately 80 and 60\%, respectively. CYP2B1 and 2B2 can be differentiated on the basis of their molecular weights, but as shown in Fig. 2c, only one microsomal protein was reactive with antibody to CYP2B1 in this study. The reasons for this phenomenon were unclear.

Because the results of western blot analysis showed that AsA deficiency decreased the amount of cytochrome P-450 immunochemically related to CYP1A1 and CYP2B2 in rats treated with PCB, to study the effects of AsA deficiency on synthesis of P-450 apoprotein, we examined liver mRNA level for CYP1A and CYP2B subfamily induced by xenobiotics. As shown in Fig. 3, the level of mRNA for CYP1A1, 1A2, and 2B1/2B2 were quantitated by RNA blot analysis using cDNA for rat CYP1A2 and CYP2B1. The TaqI-Pst I region of cDNA for CYP1A2

pyrene produced per minute per milligram microsomal protein. Statistical significance of differences among values was analyzed by ANOVA. When interaction was significant (a and $c$ ), Duncan's multiple range test was performed among group A, B, C and among D, E, F distinctively. Bars not followed by the same superscript letter (italic) are significantly different $(p<$ 0.05). Group designation is shown in Table 1. 
a

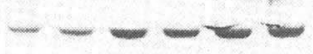

CYP2B 1/2B2

b

\section{$-$}

CYP1A1
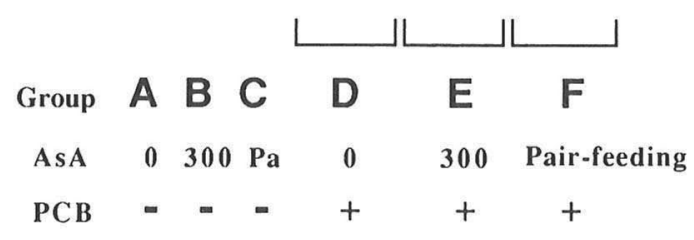

C

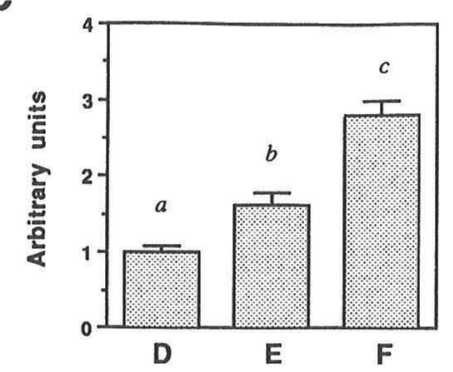

d

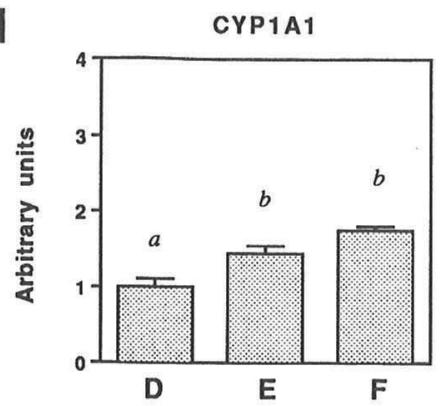

Fig. 2. Effect of AsA deficiency on the forms of cytochrome P-450 immunochemically related to CYP2B1 and CYP1A1. a and b: Western blot analysis of liver microsomes with antibodies to rat CYP2B1 and CYP1A1 was performed. SDS polyacrylamide gel electrophoriesis and Western blotting of liver microsomes obtained from one or two rats in each group were carried out as described in experimental procedures. $\mathrm{c}$ and $\mathrm{d}$ : Intensities of bands were measured by means of a densitometer. Arbitrary unit is expressed as relative unit for group D. Statistical significance of differences among values was analyzed by Duncan's multiple range test. Bars not followed by the same superscript letter (italic) are significantly different $(p<0.05)$. Group designation is shown in Table 1 .

used in this study is cross-hybridized with both CYP1A1 and 1A2 mRNA (27), and the mRNA of CYP1A1 and 1A2 can be differentiated by agarose gel electrophoresis on the basis of their molecular weights. Thus, they can be detected simultaneously. On the other hand, there is 97\% homology between CYP2B1 mRNA and 2B2 mRNA, so the cDNA for CYP2B1 used in this study cannot differentiate between them. Therefore, we detected the mRNA of the two phenobarbitalinducible cytochrome P-450 as CYP2B1+2B2 (CYP2B1/2B2). In rats without dietary PCB (group A, B, C) the mRNAs are not observed. Both CYP1A and 

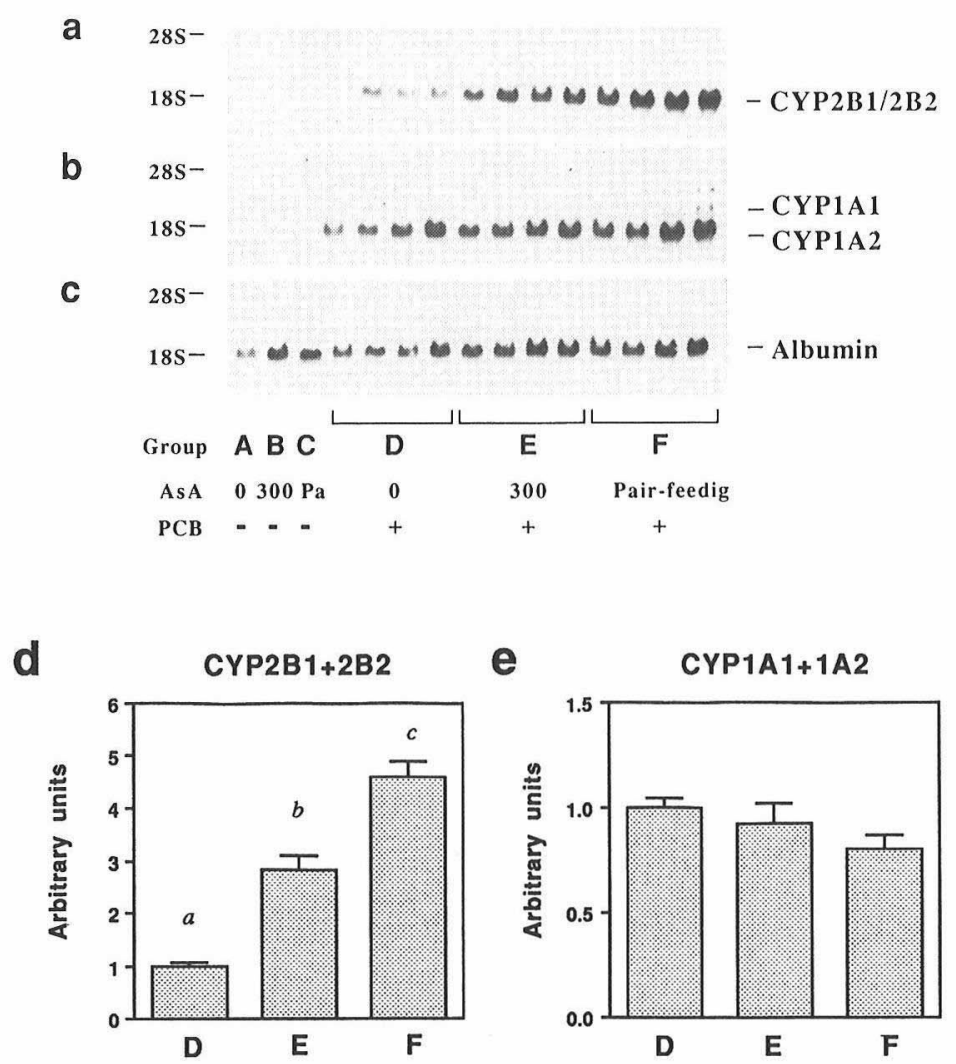

Fig. 3. Northern blot analysis of liver CYP2B1/2B2 and CYP1A1/1A2 mRNA in AsA-deficient rats $(\mathrm{a}-\mathrm{c})$, and quantitation of mRNA level by slot blot analysis (d and e). a and d: CYP2B1/2B2; b and e: CYP1A1 and 1A2; c: albumin. Arbitrary unit is expressed as relative unit for group D. Statistical significance of differences among values was analyzed by Duncan's multiple range test. Bars not followed by the same superscript letter (italic) are significantly diffrent $(p<$ 0.05 ). Group designation is shown in Table 1.

CYP2B were induced only in rats fed PCB (group D, E, F). Figure 3b, c show the levels of mRNA quantitated by RNA dot blot analysis. AsA deficiency decreased the level of CYP2B1/2B2 mRNA induced by dietary PCB about 40\%. Pair-feeding increased CYP2B1/2B2 mRNA. However, AsA deficiency did not affect the level of CYP1A1 and CYP1A2 mRNA, and pair-feeding had no effect on them either. As mentioned above, dietary AsA showed a differential effect on the mRNA level of individual cytochrome P-450 induced by PCB. 


\section{DISCUSSION}

Multiple forms of cytochrome P-450 are present in liver microsomes of animals and some of them are inducible in different ways by administration of various kinds of chemical compounds (1-3). The xenobiotics-inducible forms of cytochrome P-450 catalyze the oxidative metabolism of a wide variety of exogenous compounds. In the present study we divided them into two types, MC-inducible forms (CYP1A1 and CYP1A2) and PB-inducible forms (CYP2B1 and CYP2B2). Each of them is markedly induced by $\mathrm{MC}$ or $\mathrm{PB}$, respectively, but in a distinct manner (1-3). On the other hand, PCB markedly induces both of them in an unknown manner. Because our purpose in this study was surveying a specific forms of cytochrome P-450 that is very sensitive to AsA deficiency, we selected PCB as an inducer.

In agreement with our previous reports (9), the concentration of total cytochrome P-450 decreased in AsA-deficient rats with and without dietary PCB (Fig. 1a). The activities of aminopyrine-N-demethylase caused a decrease in AsAdeficient rats (Fig. 1b), whereas the activities of benzo(a)pyrene hydroxylase were not affected by AsA deficiency (Fig. 1c). These data suggested that the specific forms of cytochrome P-450 would be susceptible to AsA deficiency. The benzo(a)pyrene hydroxylase activity is mainly catalyzed by the members of the CYP1A subfamily, especially CYP1A1 $(2,3)$. It is likely that CYP1A subfamily is not affected by AsA deficiency, and that specific forms of cytochrome P-450 which have an aminopyrine- $\mathrm{N}$-demethylase activity are very sensitive to AsA deficiency.

Western blot analyses were aimed at investigating the influence of AsA deficiency on the protein amounts of each form of cytochrome P-450 induced by dietary PCB. In rats fed PCB, the amounts of cytochrome P-450 that are immunochemmically reactive with antibodies to CYP1A1 and CYP2B1 significantly increased. Both forms were decreased in AsA-deficient rats, but the degree of reduction of cytochrome P-450 related to CYP2B1 was greater than CYP1A1 (Fig. 2). It seems reasonable to suppose that the amounts of PB-inducible forms are quite susceptible to AsA deficiency.

We performed RNA blot analysis to investigate whether dietary AsA influences the inducible expression of cytochrome P-450 gene by PCB (Fig. 3). AsA deficiency reduced the hepatic level of CYP2B1/2B2 mRNA induced by PCB, but had no effect on the abundance of CYP1A1 and CYP1A2 mRNA. These results indicated that dietary AsA affected gene expression of cytochrome P-450, and that AsA deficiency caused a selective reduction in the level of mRNA for PB-inducible CYP2B1/2B2 among cytochrome P-450 induced by PCB. From these three assays (i.e., drug-metabolizing enzyme activity, western blot analysis and RNA blot analysis) we concluded that AsA deficiency decreases specific forms of xenobioticsinducible cytochrome P-450, especially CYP2B1/2B2, and that the reduction of CYP2B1/2B2 mRNA level in AsA-deficient rats caused a decrease in the concentra- 
tion of cytochrome P-450 and hepatic activities of MFO.

In the case of CYP1A1, the protein amount was decreased but the level of mRNA not changed in AsA deficiency. In this study PCB was continuously supplied for 15 days. Taking the turn over of mRNA into consideration, it may be necessary to investigate the time course change of the level of CYP1A1 mRNA toward a better understanding of the effect of AsA on CYP1A subfamily.

Restriction of feeding increased the concentration of total cytochrome P-450 (nmol/mg microsomal protein), the activities of aminopyrine- $\mathrm{N}-$ demethylase and the level of CYP2B1/2B2 mRNA, contrary to our expectations (Figs. 1-3). One explanation for this phenomenon may be that hormonal changes or other metabolic changes caused by pair-feeding increased the concentration of total cytochrome $\mathbf{P}$ 450. It is known that fasting increases the level of some forms of cytochrome P-450 (30). However, we have no definite information on the increase in cytochrome P450 by pair-feeding.

AsA deficiency decreased the concentration of total cytochrome P-450 and the activity of aminopyrine-N-demethylase in rats even without PCB (Fig, 1a, b). The effect of dietary AsA on other forms of cytochrome P-450 is also worth noting. Our previous reports showed that AsA deficiency decreased an activity of cholesterol$7 \alpha$-hydroxylase (EC 1.14.13.17) in ODS rats (31). Kanazawa et al. (14) showed that CYP1A2 and CYP2E1 decreased in AsA-deficient guinea pigs. These data support the explanation that dietary AsA also an important role in the maintenance of other forms of cytochrome P-450 constitutively expressed in liver or other organs.

In the present study we demonstrated that dietary AsA selectively influenced the level of mRNA for CYP2B1/2B2 induced by PCB. The molecular mechanism of AsA function through the expression of CYP2B1/2B2 gene should be the next target for our investigation. An induction of these cytochrome P-450 is directly mediated by xenobiotics itself (1-3), therefore the level of these cytochrome P-450 mRNAs increased markedly in rats fed PCB. It seems that dietary AsA influences optimum induction of cytochrome P-450 by xenobiotics. Our interest is in understanding whether AsA affects the mRNA level transcriptionally or posttranscriptionally, either directly or indirectly.

It is likely that the experiments indicated as follows would be suggestive in discussion of the AsA function in detail. First, it seems that PB would be much effective as an inducer in discussion of the expression of CYP2B1/2B2 gene. PCB may be effective in surveying the effect of AsA on many forms of cytochrome P450 , but possibly not effective in investigating the biochemical function of AsA agaisnt the induction of a specific form of cytochrome P-450 hereafter. Secondly, we wish to analyze the level of mRNA accumulating in the short term, to study the possibility that dietary AsA may influence the synthesis of CYP2B1/2B2 de novo. In the present study, rats were continuously treated with PCB for an experimental period of 15 days. It is well known, however, that the induction of CYP2B1/2B2 by $P B$ is caused by transcriptional activation, and the maximal accumulation of 
mRNAs can be found in the short term $(2,3)$. Thirdly, our next target is to investigate the exact role of AsA for the maintenance and induction of cytochrome P-450 on molecular basis using cultured hepatocytes. Bissell and Guzelian (32) demonstrated that the supplementation of the culture medium with AsA at a concentration sufficient to restore intracellular levels of AsA to normal resulted in maintenance of significantly increased concentration of cytochrome P-450. This observation shows that AsA may directly function at hepatocyte for maintenance of cytochrome P-450. Fourthly, the availability of heme for cytochrome P-450 synthesis has been shown to be unchanged by AsA deficiency $(33,34)$. We also observed that intraperitoneal injection of aminolevulinic acid, the key substrate for heme synthesis, neither prevented nor recovered the cytochrome P-450 content and the enzyme activities in ODS rats (Kobayashi, Horio, and Yoshida, unpublished data). These date suggest that the AsA deficiency does not affect the availability of heme for cytochrome P-450 synthesis. However, it can be supposed that AsA deficiency alters the reduced status of heme, or affects the association of heme with apoprotein. Therefore, there is room for further investigation with regard to the relationships between AsA deficiency and the synthesis or association of heme.

We believe that elucidation of the biochemical function of AsA for the expression of cytochrome P-450 will facilitate an understanding of a novel nutritional and physiological significance of AsA.

The authors are grateful to Dr. K. Kawajiri (Saitama Cancer Center Research Institute), Dr. Y. Fujii-Kuriyama (Tohoku University), and Dr. K. Nakamura (Nagoya University) for cDNA probe against CYP1A2, cDNA probe against CYP2B1, and cDNA probe against rat albumin, respectively. This work was suported in part by a grant from the Elizabeth Arnold Fuji Foundation, Japan.

\section{REFERENCES}

1) Lu, A. Y. H., and West, S. B. (1980): Multiplicity of mammalian microsomal cytochromes P-450. Pharmacol. Rev., 31, 277-295.

2) Whitlock, J. P., Jr. (1986): The regulation of cytochrome P-450 gene expression. Annu. Rev. Pharmacol. Toxicol., 26, 333-369.

3) Nebert, D. W., and Gonzalez, F. J. (1987): P-450 genes: Structure, evolution and regulation. Annu. Rev. Biochem., 56, 945-993.

4) Kato, R. (1977): Drug metabolism under pathological and abnormal physiological states in animals and man. Xenobiotica, 7, 25-92.

5) Hathcock, J. N. (1985): Metabolic mechanisms of drug-nutrient interactions. Fed. Proc., 44, 124-129.

6) Zannoni, V. G., Flynn, E. J., and Lynch, M. (1972): Ascorbic acid and drug metabolism. Biochem. Pharmacol., 21, 1377-1392.

7) Zannoni, V. G., and Lynch, M. (1973): The role of ascorbic acid in drug metabolism. Drug Metab. Rev., 2, 57-69.

8) Skic, B. I., Mimnauhgh, E. G., Litterest, C. L., and Gram, T. E. (1977): The effects 
of ascorbic acid deficiency and repletion on pulmonary, renal, and hepatic drug metabolism in the drug metabolism. Arch. Biochem. Biophys., 179, 663-671.

9) Horio, F., Ozaki, K., Kohmura, M., Yoshida, A., Makino, S., and Hayashi, Y. (1986): Ascorbic acid requirement for the induction of microsomal drug-metabolizing enzymes in a rat mutant unable to synthesize ascorbic acid. J. Nutr., 116, 2278-2289.

10) Myllyla, R., Kuitti-Savadainen, E. R., and Kivirikko, K. I. (1978): The role of ascorbate in the prolyl hydroxylase reaction. Biochem. Biophys. Res. Commun., 83, 441-448.

11) Makino, S., and Katagiri, K. (1980): Osteogenic disorder rat. Exp. Anim. (Jpn.), 29, 374-375.

12) Horio, F., and Yoshida, A. (1982): Effects of some xenobiotics on ascorbic acid metabolism in rats. J. Nutr., 112, 416-425.

13) Horio, F., Kimura, M., and Yoshida, A. (1983): Effect of several xenobiotics on the activities of enzymes affecting ascorbic acid synthesis in rats. J. Nutr. Sci. Vitaminol., 29, 233-247.

14) Kanazawa, Y., Kitada, M., Mori, T., Inukai, Y., Imaoka, S., Funae, Y., and Kamataki, T. (1991): Ascorbic acid deficiency decreases specific forms of cytochrome P-450 in liver microsomes of guinea pigs. Mol. Pharmacol., 39, 456-460.

15) American Institute of Nutrition (1977): Report of the American Institute of Nutrition ad hoc committee on standards for nutritional studies. J. Nutr., 107, 1340-1348.

16) American Institute of Nutrition (1980): Second report of the ad hoc committee on standards for nutritional studies. J. Nutr., 110, 1726.

17) Horio, F., Ozaki, K., Yoshida, A., Makino, S., and Hayashi, Y. (1985): Requirement for ascorbic acid in a rat mutant unable to synthesize ascorbic acid. J. Nutr., 115, $1630-1640$.

18) Kato, N., Tani, T., and Yoshida, A. (1980): Effect of dietary level of protein on liver microsomal drug-metabolizing enzymes, urinary ascorbic acid and lipid metabolism in rats fed PCB-containing diets. J. Nutr., 110, 1689-1694.

19) Omura, T., and Sato, R. (1964): The carbon monooxide binding pigment of liver microsomes. J. Biol. Chem., 239, 2370-2378.

20) Nash, T. (1953): The colorimetric estimation of formaldehyde by means of Hantzch reaction. Biochem. J., 55, 416-421.

21) Nebert, D. W., and Gelboin, H. (1968): Substrate-inducible microsomal aryl hydroxylase in mammalian cell culture. J. Biol. Chem., 243, 6242-6249.

22) Laemmli, U. K. (1970): Cleavage of structural proteins during the assembly of the head of bacteriophage T4. Nature, 227, 680-685.

23) Chomozynski, P., and Sacchi, N. (1987): Single-step method of RNA isolation by guanidium thiocyanate-phenol-chloroform extraction. Anal. Biochem., 162, 156-159.

24) Thomas, P. (1980): Hybridization of denatured RNA and small DNA fragments transferred to nitrocellulose. Proc. Natl. Acad. Sci. U.S.A., 77, 5201-5205.

25) Southern, E. M. (1975): Detection of specific sequences among DNA fragments separated by gel electrophoresis. J. Mol. Biol., 98, 503-517.

26) Kawajiri, K., Gotoh, O., Sogawa, K., Tagashira, Y., Muramatsu, M., and FujiiKuriyama, Y. (1984): Coding nucleotide sequence of 3-methylcholanthrene-inducible cytochrome P-450d cDNA from rat liver. Proc. Natl. Acad. Sci. U.S.A., 81, 16491653. 
27) Kawajiri, K., Gotoh, O., Tagashira, Y., Sogawa, K., and Fujii-Kuriyama, Y. (1984): Titration of mRNAs for cytochrome P-450c and P-450d under drug-inductive conditions in rat livers by their specific probes of cloned DNAs. J. Biol. Chem., 259, 1014510149.

28) Fujii-Kuriyama, Y., Mizukami, Y., Kawajiri, K., Sogawa, S., and Muramatsu, M. (1982): Primary structure of a cytochrome P-450: Coding nucleotide sequence of phenobarbital-inducible cytochrome P-450 cDNA from rat liver. Proc. Natl. Acad. Sci. U.S.A., 79, 2793-2797.

29) Iwatsuki, N., Hattori, T., Iwatsuki, Y., Nakano, M., and Nakamura, K. (1987): A complete rat serum albumin cDNA clone directly identified by immunological screening of cDNA expression library. Agric. Biol. Chem., 51, 379-384.

30) Imaoka, S., Terano, Y., and Funae, Y. (1990): Changes in the amount of cytochrome P-450s in rat hepatic microsomes with starvation. Arch. Biochem. Biophys., 278, 168178.

31) Horio, F., Ozaki, K., Oda, H., Makino, S., Hayashi, Y., and Yoshida, A. (1989): Effect of dietary ascorbic acid, cholesterol and PCB on cholesterol and bile acid metabolism in a rat mutant unable to synthesize ascorbic acid. J. Nutr., 119, 409-415.

32) Bissell, D. M., and Guzelian, P. S. (1979): Ascorbic acid deficiency and cytochrome P-450 in adult rat hepatocytes in primary monolayer culture. Arch. Biochem. Biophys., 192, 569-576.

33) Rikans, L. E., Smith, C. R., and Zannoni, V. G. (1978): Ascorbic acid and cytochrome P-450. J. Pharmacol. Exp. Ther., 204, 702-713.

34) Turnbull, J. D., and Omaye, S. T. (1980): Synthesis of cytochrome P-450 heme in ascorbic acid-deficient guinia pigs. Biochem. Pharmacol., 29, 1255-1260. 